\title{
Symmetry and monotonicity of least energy solutions
}

\author{
Jaeyoung BYEON, Louis JEANJEAN ${ }^{\dagger}$ and Mihai MARIŞ ${ }^{\ddagger}$
}

\begin{abstract}
We give a simple proof of the fact that for a large class of quasilinear elliptic equations and systems the solutions that minimize the corresponding energy in the set of all solutions are radially symmetric. We require just continuous nonlinearities and no cooperative conditions for systems. Thus, in particular, our results cannot be obtained by using the moving planes method. In the case of scalar equations, we also prove that any least energy solution has a constant sign and is monotone with respect to the radial variable. Our proofs rely on results in $[14,6]$ and answer questions from $[3,11]$.
\end{abstract}

\section{Introduction}

We consider the system of partial differential equations

$$
-\operatorname{div}\left(\left|\nabla u_{i}\right|^{p-2} \nabla u_{i}\right)=g_{i}(u), \quad i=1, \ldots, m
$$

where $u=\left(u_{1}, \ldots, u_{m}\right): \mathbf{R}^{N} \longrightarrow \mathbf{R}^{m}, 1<p<\infty,\left|\left(y_{1}, \ldots, y_{N}\right)\right|^{p}=\left(\sum_{j=1}^{N} y_{j}^{2}\right)^{\frac{p}{2}}$, $g_{i}(0)=0$ and there exists $G \in C^{1}\left(\mathbf{R}^{m} \backslash\{0\}, \mathbf{R}\right) \cap C\left(\mathbf{R}^{m}, \mathbf{R}\right)$ such that $g_{i}(u)=\frac{\partial G}{\partial u_{i}}(u)$ for $u \neq 0$.

Formally, solutions of (1) are critical points of the following energy functional

$$
S(u)=\frac{1}{p} \int_{\mathbf{R}^{N}} \sum_{i=1}^{m}\left|\nabla u_{i}\right|^{p} d x-\int_{\mathbf{R}^{N}} G(u) d x .
$$

The aim of this note is to prove, under general assumptions, that those solutions of (1) which minimize the energy $S$ in the set of all solutions are radially symmetric (up to a translation in $\mathbf{R}^{N}$ ). In the scalar case we also study the sign and monotonicity of these solutions. We do not consider here the problem of existence of solutions (respectively of least energy solutions) for (1). We believe that our results cover all situations where the existence of a least energy solution is already known in the literature.

We begin with some definitions. Let $\Pi$ be an affine hyperplane in $\mathbf{R}^{N}$, let $\Pi^{+}$and $\Pi^{-}$be the two closed half-spaces determined by $\Pi$ and $s_{\Pi}$ the symmetry with respect

\footnotetext{
${ }^{*}$ Department of Mathematics and PMI, Pohang University of Science and Technology, Pohang, Kyungbuk 790-784, Republic of Korea, e-mail: jbyeon@postech.ac.kr

†Département de Mathématiques UMR 6623, Université de Franche-Comté, 16, Route de Gray, 25030 Besançon, France, e-mail: louis.jeanjean@univ-fcomte.fr

${ }^{\ddagger}$ Département de Mathématiques UMR 6623, Université de Franche-Comté, 16, Route de Gray, 25030 Besançon, France, e-mail: mihai.maris@univ-fcomte.fr
} 
to $\Pi$ (i.e. $s_{\Pi}(x)=2 p_{\Pi}(x)-x$, where $p_{\Pi}$ is the orthogonal projection onto $\Pi$ ). Given a function $f$ defined on $\mathbf{R}^{N}$, we define

$$
f_{\Pi^{+}}(x)=\left\{\begin{array}{ll}
f(x) & \text { if } x \in \Pi^{+} \\
f\left(s_{\Pi}(x)\right) & \text { if } x \in \Pi^{-}
\end{array}, \quad f_{\Pi^{-}}(x)= \begin{cases}f(x) & \text { if } x \in \Pi^{-} \\
f\left(s_{\Pi}(x)\right) & \text { if } x \in \Pi^{+}\end{cases}\right.
$$

For $\sigma>0$, we denote $f_{\sigma}(x)=f\left(\frac{x}{\sigma}\right)$. We say that a space $\mathcal{X}$ of functions defined on $\mathbf{R}^{N}$ is admissible if $\mathcal{X}$ is nonempty and

(i) $\mathcal{X} \subset L_{l o c}^{1}\left(\mathbf{R}^{N}, \mathbf{R}^{m}\right)$ and measure $(\{x|| u(x) \mid>\alpha\})<\infty$ for any $u \in \mathcal{X}$ and $\alpha>0$;

(ii) $g_{i}(u) \in L_{l o c}^{1}\left(\mathbf{R}^{N}\right)$ for any $u \in \mathcal{X}$ and $i=1, \ldots, m$;

(iii) $\sum_{i=1}^{m}\left|\nabla u_{i}\right|^{p}$ and $G(u)$ belong to $L^{1}\left(\mathbf{R}^{N}\right)$ if $u \in \mathcal{X}$;

(iv) $u_{\sigma} \in \mathcal{X}$ for any $u \in \mathcal{X}$ and $\sigma>0$;

(v) $u_{\Pi^{+}}, u_{\Pi^{-}} \in \mathcal{X}$ whenever $u \in \mathcal{X}$ and $\Pi$ is an affine hyperplane in $\mathbf{R}^{N}$.

Let $\mathcal{X}$ be an admissible function space. We note that from (i) and (iii), $G(0)=0$. A function $u \in \mathcal{X}$ is a solution of (1) if it satisfies (1) in $\mathcal{D}^{\prime}\left(\mathbf{R}^{N}\right)$. If (1) admits solutions in $\mathcal{X}$, we say that $\underline{u}$ is a least energy solution if $\underline{u}$ is a nontrivial solution of (1) and

$$
S(\underline{u})=\inf \{S(u) \mid u \in \mathcal{X} \backslash\{0\}, u \text { is a solution of }(1)\} .
$$

We introduce the functionals

$$
J(u)=\frac{1}{p} \int_{\mathbf{R}^{N}} \sum_{i=1}^{m}\left|\nabla u_{i}\right|^{p} d x \quad \text { and } \quad V(u)=\int_{\mathbf{R}^{N}} G(u) d x .
$$

Clearly, these functionals are well-defined on any admissible function space. As we will see, the least energy solutions of (1) come from the following minimization problem:

$$
\text { minimize } J(u) \text { in the set }\{u \in \mathcal{X} \mid V(u)=\lambda\} \text {. }
$$

We shall prove that under some general conditions (see (C1)-(C3) or (D1)-(D3) below), all least energy solutions of (1) in the set $\mathcal{X}$ are radially symmetric, up to a translation in $\mathbf{R}^{N}$.

It is easy to see that $J\left(u_{\sigma}\right)=\sigma^{N-p} J(u)$ and $V\left(u_{\sigma}\right)=\sigma^{N} V(u)$. If $V(u)>0$ for some $u \in \mathcal{X}$, we have $V\left(u_{\sigma}\right)=1$ for $\sigma=V(u)^{-\frac{1}{N}}$. Then, denoting

$$
T=\inf \{J(u) \mid u \in \mathcal{X} \text { and } V(u)=1\}
$$

we see that

$$
J(v) \geq T(V(v))^{\frac{N-p}{N}} \quad \text { for any } v \in \mathcal{X} \text { satisfying } V(v)>0 .
$$

It is clear that $u$ is a minimizer for problem $\left(\mathcal{P}_{\lambda}\right)$ above $(\lambda>0)$ if and only if $u_{\sigma_{1}}$ is a minimizer for $\left(\mathcal{P}_{1}\right)$, where $\sigma_{1}=\lambda^{-\frac{1}{N}}$.

We assume first that $1<p<N$ and the following conditions are satisfied.

(C1) $T>0$ and problem $\left(\mathcal{P}_{1}\right)$ has a minimizer $u_{*} \in \mathcal{X}$; 
(C2) Any minimizer $u \in \mathcal{X}$ of $\left(\mathcal{P}_{1}\right)$ is a $C^{1}$ function and satisfies the Euler-Lagrange system of equations

$$
-\operatorname{div}\left(\left|\nabla u_{i}\right|^{p-2} \nabla u_{i}\right)=\alpha g_{i}(u) \quad \text { in } \mathcal{D}^{\prime}\left(\mathbf{R}^{N}\right)
$$

for $i=1, \ldots, m$ and some $\alpha \in \mathbf{R}$;

(C3) Any solution $u \in \mathcal{X}$ of (4) (and not only any minimizer!) satisfies the Pohozaev identity

$$
(N-p) J(u)=\alpha N V(u) .
$$

A few comments are in order. Clearly, the most important of the conditions above is (C1). To our knowledge, the existence of a minimizer for $\left(\mathcal{P}_{1}\right)$, under sufficiently general assumptions on the functions $g_{i}$ and for arbitrary $m \in \mathbf{N}^{*}$ and $p \in(1, \infty)$, is still an open problem. However, several particular cases have been extensively studied in the literature. A series of papers has been devoted to the case $p=2$ and fairly optimal conditions on $g_{i}$ that guarantee (C1) have been found by Berestycki-Lions [1] for $m=1$ and by Brezis-Lieb [3] for $m \geq 1$. In the case $m=1$ and $1<p<N$ the existence of a minimizer for $\left(\mathcal{P}_{1}\right)$ has also been proved in [9] under general assumptions on $g=g_{1}$ (similar to the assumptions in [1]). Under the conditions considered in [1] and [9], the functionals $J$ and $V$ are well defined on $W^{1, p}\left(\mathbf{R}^{N}\right)$ and this is clearly an admissible function space. The setting in [3] also corresponds to our assumptions.

If $T>0$ and $\left(\mathcal{P}_{1}\right)$ admits minimizers, in most applications it is quite standard to prove that (C2) and (C3) hold. This is indeed the case under the assumptions in $[1,3,9]$.

Next we consider the case $p=N$. Note that in this case the Pohozaev identity (5) becomes $\alpha N V(u)=0$; hence any "reasonable" solution $u$ of (1) should satisfy $V(u)=0$. Since we are interested in nontrivial solutions, we consider the minimization problem

$$
\text { minimize } J(u) \text { in the set }\{u \in \mathcal{X} \backslash\{0\} \mid V(u)=0\} .
$$

We assume that the following conditions are satisfied.

(D1) $T_{0}:=\inf \{J(u) \mid u \in \mathcal{X}, u \neq 0, V(u)=0\}>0$ and $\left(\mathcal{P}_{0}^{\prime}\right)$ admits a minimizer $u_{0}$;

(D2) Any minimizer $u \in \mathcal{X}$ of $\left(\mathcal{P}_{0}^{\prime}\right)$ is $C^{1}$ and satisfies the Euler-Lagrange equations (4) for some $\alpha>0$;

(D3) Any solution $u \in \mathcal{X}$ of (4) (with $\alpha>0$ ) satisfies the Pohozaev identity $V(u)=0$.

For $p=N=2$, fairly optimal conditions on $g_{i}$ that guarantee (D1)-(D3) have been found by Berestycki-Gallouët-Kavian [2] for $m=1$ and by Brezis-Lieb [3] for $m \geq 1$.

In the next section we show that least energy solutions are minimizers of $\left(\mathcal{P}_{\lambda}\right)$ for some particular choice of $\lambda$ if $1<p<N$, respectively minimizers of $\left(\mathcal{P}_{0}^{\prime}\right)$ if $p=N$. Then we obtain the radial symmetry of such solutions as a direct consequence of the general results in [14] (in the case $N=p$, we need some extra-argument in addition to the results in [14].

In the third section we consider the scalar case $m=1$ and we prove that least energy solutions have constant sign and, if they tend to zero at infinity, then they are monotone with respect to the radial variable.

In the final section we make some connections with related results of symmetry and monotonicity in the literature. Let us just mention that, especially in the scalar case, the symmetry and monotonicity of solutions of (1) have been studied by many authors, see 
e.g. $[10,15,8,7]$ and references therein. However, in all these works it is assumed that the solutions are nonnegative and some further assumptions on the nonlinearity $g$ are made. They require, at least, $g$ to be Lipschitz continuous and to satisfy a cooperative condition in the case of systems. In the present work, we do not make any additional assumptions on $g$, except those that guarantee the existence of least energy solutions (basically, we need $g$ to be merely continuous and to satisfy some growth conditions near zero, see [3] and [9]). We prove that our solutions have constant sign and our results are valid as well for compactly supported solutions and for solutions that do not vanish. Of course, there is a price we have to pay: our method works only for least energy solutions, not for any nonnegative solution of (1).

\section{Variational characterization and symmetry}

We begin with the case $1<p<N$.

Lemma 1 Assume that $1<p<N$ and the conditions (C1)-(C3) hold.

(i) Let $u$ be a minimizer for $\left(\mathcal{P}_{1}\right)$. Then $u_{\sigma_{0}}$ is a least action solution of (1), where $\sigma_{0}=\left(\frac{N-p}{N} T\right)^{\frac{1}{p}}$, and $S\left(u_{\sigma_{0}}\right)=p(N-p)^{\frac{N}{p}-1} N^{-\frac{N}{p}} T^{\frac{N}{p}}$.

(ii) Let $v$ be a least energy solution for (1). Then $v$ is a minimizer for $\left(\mathcal{P}_{\lambda}\right)$, where $\lambda=\left(\frac{N-p}{N} T\right)^{\frac{N}{p}}$.

Proof. (i) By (C2) we know that $u \in C^{1}$ and $u$ satisfies (4) for some $\alpha \in \mathbf{R}$. Then (5) implies $(N-p) J(u)=\alpha N V(u)$, which gives $\alpha=\frac{N-p}{N} T>0$. It is easy to see that $u_{\sigma_{0}}$ satisfies (1) for $\sigma_{0}=\alpha^{\frac{1}{p}}$ and

$$
S\left(u_{\sigma_{0}}\right)=\sigma_{0}^{N-p} J(u)-\sigma_{0}^{N} V(u)=\sigma_{0}^{N-p} T-\sigma_{0}^{N}=p(N-p)^{\frac{N}{p}-1} N^{-\frac{N}{p}} T^{\frac{N}{p}} .
$$

Let $w \in \mathcal{X}, w \neq 0$, be a solution of (1). By (C3) we have $(N-p) J(w)=N V(w)$. If $J(w)=0$, we have $\nabla w=0$ a.e. on $\mathbf{R}^{N}$, hence $w$ must be constant. Since measure $\{x \in$ $\left.\mathbf{R}^{N}|| w(x)>\alpha\right\}<\infty$ for any $\alpha>0$, we infer that $w=0$, a contradiction. Thus $J(w)>0$ and $V(w)=\frac{N-p}{N} J(w)>0$. On the other hand, by (3) we get $J(w) \geq T(V(w))^{\frac{N-p}{N}}$, i.e. $J(w) \geq T\left(\frac{N-p}{N} J(w)\right)^{\frac{N-p}{N}}$, which gives

$$
J(w) \geq\left(\frac{N-p}{N}\right)^{\frac{N-p}{p}} T^{\frac{N}{p}} .
$$

Combined with Pohozaev identity, this implies

$$
S(w)=J(w)-V(w)=\frac{p}{N} J(w) \geq p(N-p)^{\frac{N}{p}-1} N^{-\frac{N}{p}} T^{\frac{N}{p}}=S\left(u_{\sigma_{0}}\right)
$$

and we infer that $u_{\sigma_{0}}$ is a least energy solution for (1).

(ii) Conversely, let $v$ be a least energy solution for (1). Then $(N-p) J(v)=N V(v)$ by (C3), hence $S(v)=\frac{p}{N} J(v)$. It is obvious that the inequalities (6) and (7) above are satisfied with $w=v$. On the other hand, $S(v)=S\left(u_{\sigma_{0}}\right)$ and we infer that $v$ must satisfy (7) with equality sign, that is,

$$
J(v)=\left(\frac{N-p}{N}\right)^{\frac{N-p}{p}} T^{\frac{N}{p}} \text { and } V(v)=\frac{N-p}{N} J(v)=\left(\frac{N-p}{N}\right)^{\frac{N}{p}} T^{\frac{N}{p}} .
$$


A simple scaling argument shows that $v$ is a minimizer for $\left(\mathcal{P}_{\lambda}\right)$, where $\lambda=\left(\frac{N-p}{N}\right)^{\frac{N}{p}} T^{\frac{N}{p}}$; equivalently, $v_{\sigma_{1}}$ is a minimizer for $\left(\mathcal{P}_{1}\right)$, where $\sigma_{1}=\left(\frac{N-p}{N} T\right)^{-\frac{1}{p}}=\sigma_{0}^{-1}$. This completes the proof of Lemma 1.

The symmetry of least energy solutions will follow from Lemma 1 and a general symmetry result in [14]. For the convenience of the reader, we recall here that result.

Theorem 2 ([14]) Assume that $u: \mathbf{R}^{N} \longrightarrow \mathbf{R}^{m}$ belongs to some function space $\mathcal{Y}$ and solves the minimization problem

$$
\begin{aligned}
& \text { minimize } \int_{\mathbf{R}^{N}} F(u(x),|\nabla u(x)|) d x \\
& \text { in the set }\left\{u \in \mathcal{Y} \mid \int_{\mathbf{R}^{N}} H(u(x),|\nabla u(x)|) d x=\lambda \neq 0\right\} .
\end{aligned}
$$

Suppose that the following conditions are satisfied:

(A1) For any $v \in \mathcal{Y}$ and any affine hyperplane $\Pi$ in $\mathbf{R}^{N}$ we have $v_{\Pi^{+}}, v_{\Pi^{-}} \in \mathcal{Y}$.

(A2) Problem $(\mathcal{P})$ admits minimizers in $\mathcal{Y}$ and any minimizer is a $C^{1}$ function on $\mathbf{R}^{N}$.

Then, after a translation, $u$ is radially symmetric.

Lemma 1 implies that least energy solutions solve the minimization problem $\left(\mathcal{P}_{\lambda}\right)$ for some $\lambda>0$. Conditions (C1), (C2) and property (v) in the definition of admissible spaces imply that $\left(\mathcal{P}_{\lambda}\right)$ satisfies the assumptions of Theorem 2. Thus we get:

Proposition 3 Assume that $1<p<N$ and (C1)-(C3) hold. Then (1) admits a least energy solution and each least energy solution is radially symmetric (up to a translation in $\mathbf{R}^{N}$ ).

Now we turn our attention to the case $p=N$.

Proposition 4 Assume that $p=N$ and (D1)-(D3) hold. Then (1) admits a least energy solution and any least energy solution solves $\left(\mathcal{P}_{0}^{\prime}\right)$.

Moreover, if we assume that $G$ is either negative or positive in some ball $B_{\mathbf{R}^{m}}(0, \varepsilon) \backslash$ $\{0\}$ and $u \in \mathcal{X}$ is a least energy solution such that $u(x) \longrightarrow 0$ as $|x| \longrightarrow \infty$, then $u$ is radially symmetric (up to a translation in $\mathbf{R}^{N}$ ).

Proof. Let $u_{0}$ be a minimizer for $\left(\mathcal{P}_{0}^{\prime}\right)$. By (D2) and (D3) we have $V\left(u_{0}\right)=0$ and $u_{0}$ satisfies (4) for some $\alpha>0$. Let $u_{1}=\left(u_{0}\right)_{\sigma}$, where $\sigma=\alpha^{\frac{1}{p}}$. It is easy to see that $u_{1}$ solves (1) and $S\left(u_{1}\right)=J\left(u_{1}\right)-V\left(u_{1}\right)=J\left(u_{0}\right)-\sigma^{N} V\left(u_{0}\right)=J\left(u_{0}\right)=T_{0}$. For any solution $u \in \mathcal{X}, u \neq 0$ of (1) we have $V(u)=0$ by (D3) and $S(u)=J(u) \geq T_{0}=J\left(u_{1}\right)$. Hence $u_{1}$ is a least energy solution.

If $v$ is a least energy solution, then $V(v)=0$ by (D3) and $J(v)=S(v)=S\left(u_{1}\right)=T_{0}$, thus $v$ solves $\left(\mathcal{P}_{0}^{\prime}\right)$.

Although Theorem 2 does not apply directly to minimizers of problem $\left(\mathcal{P}_{0}^{\prime}\right)$ (because the value of the constraint in $\left(\mathcal{P}_{0}^{\prime}\right)$ is zero), its proof can still be adapted to those minimizers. Indeed, the proof of Theorem 2 shows that whenever $u$ is a minimizer of $(\mathcal{P})$ and $\Pi$ is an affine hyperplane such that $u_{\Pi^{+}}$and $u_{\Pi^{-}}$are also minimizers, $u$ must be symmetric with respect to $\Pi$. The only place where the assumption $\lambda \neq 0$ is used in Theorem 2 is 
to show that for any $e \in S^{N-1}$ there exists an affine hyperplane $\Pi$ orthogonal to $e$ such that

$$
\int_{\Pi^{-}} H(u(x),|\nabla u(x)|) d x=\int_{\Pi^{+}} H(u(x),|\nabla u(x)|) d x=\frac{\lambda}{2} .
$$

From (8) it follows then easily that $u_{\Pi^{+}}$and $u_{\Pi^{-}}$are also minimizers.

In the present case we will use the fact that $G(u)$ has a constant sign in a neighborhood of $\infty$ to find hyperplanes that "split the constraint in two equal parts." A similar idea has already been used in [13]. Henceforth we assume that $u$ is a least action solution, $u(x) \longrightarrow 0$ as $|x| \longrightarrow \infty$ and, say, $G(\xi)<0$ for $0<|\xi|<\varepsilon$. For $e \in S^{N-1}$ and $t \in \mathbf{R}$, we denote $\Pi_{e, t}=\left\{x \in \mathbf{R}^{N} \mid x \cdot e=t\right\}, \Pi_{e, t}^{-}=\left\{x \in \mathbf{R}^{N} \mid x \cdot e<t\right\}$ and $\Pi_{e, t}^{+}=\left\{x \in \mathbf{R}^{N} \mid x \cdot e>t\right\}$. We claim that for any $e \in S^{N-1}$, there exists $t_{e} \in \mathbf{R}$ such that

$$
\int_{\Pi_{e, t_{e}}^{-}} G(u(x)) d x=\int_{\Pi_{e, t_{e}}^{+}} G(u(x)) d x=0 \quad \text { and } \quad u_{\Pi_{e, t_{e}}^{-}} \not \equiv 0, u_{\Pi_{e, t_{e}}^{+}} \not \equiv 0 .
$$

To see this, fix $e \in S^{N-1}$ and define $\varphi_{e}^{ \pm}(t)=\int_{\Pi_{e, t}^{ \pm}} G(u(x)) d x$, respectively. It follows that $\varphi_{e}^{+}$and $\varphi_{e}^{-}$are continuous because $G(u) \in L^{1}\left(\mathbf{R}^{N}\right)$. Since $u$ is continuous, $u \not \equiv 0$, $\lim _{|x| \rightarrow \infty} u(x)=0$ and $G<0$ on $B_{\mathbf{R}^{m}}(0, \varepsilon) \backslash\{0\}$, it is not hard to see that there exist $t^{-}, t^{+} \in \mathbf{R}, t^{-}<t^{+}$such that

$$
\varphi_{e}^{-}\left(t^{-}\right)<0, \quad \varphi_{e}^{+}\left(t^{+}\right)<0 \quad \text { and } \quad u_{\Pi_{e, t^{-}}^{-}} \neq 0, \quad u_{\Pi_{e, t^{+}}^{+}} \neq 0
$$

Since $\varphi_{e}^{+}\left(t^{-}\right)=V(u)-\varphi_{e}^{-}\left(t^{-}\right)=-\varphi_{e}^{-}\left(t^{-}\right)$, it follows that $\varphi_{e}^{+}\left(t^{+}\right)<0<\varphi_{e}^{+}\left(t^{-}\right)$. From the mean value property, we see that there exists $t_{e} \in\left(t^{-}, t^{+}\right)$satisfying (9). It is clear that $u_{\Pi_{e, t e}^{-}}, u_{\Pi_{e, t e}^{+}} \in \mathcal{X} \backslash\{0\}$ because $\mathcal{X}$ is admissible and (9) implies that $V\left(u_{\Pi_{e, t_{e}}^{-}}\right)=V\left(u_{\Pi_{e, t_{e}}^{+}}\right)=0$, hence $J\left(u_{\Pi_{e, t_{e}}^{-}}\right) \geq T_{0}, J\left(u_{\Pi_{e, t_{e}}^{+}}\right) \geq T_{0}$. On the other hand, it is easy to see that $J\left(u_{\Pi_{e, t_{e}}^{-}}\right)+J\left(u_{\Pi_{e, t_{e}}^{+}}\right) \stackrel{e, t_{e}}{=} 2 J(u)=2 T_{0}$. Thus $J\left(u_{\Pi_{e, t_{e}}^{-}}\right)=J\left(u_{\Pi_{e, t_{e}}^{+}}\right)=T_{0}$ and $u_{\Pi_{e, t_{e}}^{-}}, u_{\Pi_{e, t_{e}}^{+}}$are also minimizers for $\left(\mathcal{P}_{0}^{\prime}\right)$. Then arguing exactly as in the proof of Theorem 2 in [14], it follows that after a translation, $u$ is radially symmetric.

\section{Monotonicity results}

Throughout this section we assume that $m=1$. We consider the following additional conditions for an admissible space $\mathcal{X}$.

(vi) For any $u \in \mathcal{X}$ and $t \geq 0, s \leq 0$, we have $\min (u, t) \in \mathcal{X}$ and $\max (u, s) \in \mathcal{X}$.

(vii) If $u \in \mathcal{X}$ and $u \geq 0$ (respectively $u \leq 0$ ), then $u^{*} \in \mathcal{X}$ (respectively $-(-u)^{*} \in \mathcal{X}$ ), where $u^{*}$ is the Schwarz rearrangement of $u$.

Proposition 5 Let an admissible space $\mathcal{X}$ satisfy the condition (vi). Assume that $1<$ $p<N$ and $(\mathbf{C 1})$ holds. If $u \in \mathcal{X}$ is a solution of $\left(\mathcal{P}_{\lambda}\right)$ for some $\lambda>0$, then $u$ does not change sign.

Proof. This is a simple consequence of scaling. Indeed, let $u_{+}=\max (u, 0)$ and $u_{-}=$ $\min (u, 0)$. It is clear that $V\left(u_{+}\right)+V\left(u_{-}\right)=V(u)=\lambda$ and $J\left(u_{+}\right)+J\left(u_{-}\right)=J(u)$. If $V\left(u_{-}\right)<0$, then necessarily $V\left(u_{+}\right)>\lambda$. For $\sigma=\left(\frac{\lambda}{V\left(u_{+}\right)}\right)^{\frac{1}{N}} \in(0,1)$ we have $V\left(\left(u_{+}\right)_{\sigma}\right)=$ 
$\sigma^{N} V\left(u_{+}\right)=\lambda$ and $J\left(\left(u_{+}\right)_{\sigma}\right)=\sigma^{N-p} J\left(u_{+}\right) \leq \sigma^{N-p} J(u)<J(u)$, contradicting the fact that $u$ is a minimizer. Thus necessarily $V\left(u_{-}\right) \geq 0$. In the same way $V\left(u_{+}\right) \geq 0$, therefore $V\left(u_{-}\right), V\left(u_{+}\right) \in[0, \lambda]$. Using inequality (3) (which trivially holds if $V(v)=0$ ), we get

$$
T \lambda^{\frac{N-p}{N}}=J(u)=J\left(u_{+}\right)+J\left(u_{-}\right) \geq T V\left(u_{+}\right)^{\frac{N-p}{N}}+T V\left(u_{-}\right)^{\frac{N-p}{N}},
$$

which gives

$$
1 \geq\left(\frac{V\left(u_{+}\right)}{\lambda}\right)^{\frac{N-p}{N}}+\left(\frac{V\left(u_{-}\right)}{\lambda}\right)^{\frac{N-p}{N}} .
$$

Since $V\left(u_{+}\right)+V\left(u_{-}\right)=\lambda$, (10) implies that either $V\left(u_{+}\right)=0$ or $V\left(u_{-}\right)=0$. If $V\left(u_{-}\right)=0$ and $V\left(u_{+}\right)=\lambda$ we see that $u_{+}$satisfies the constraint and

$$
J\left(u_{+}\right)=J(u)-J\left(u_{-}\right) \leq J(u) .
$$

Since $u$ is a minimizer, we must have equality in (11) and this gives $J\left(u_{-}\right)=0$, hence $u_{-}=0$ and $u=u_{+} \geq 0$. Similarly $V\left(u_{+}\right)=0$ implies $u=u_{-} \leq 0$.

Proposition 6 Let an admissible space $\mathcal{X}$ satisfy the condition (vi). Assume that $p=N$ and (D1) holds. We have:

(a) if $G<0$ on $[-\varepsilon, 0) \cup(0, \varepsilon]$ for some $\varepsilon>0$, then $u \in \mathcal{X}$ is a minimizer of ( $\left.\mathcal{P}_{0}^{\prime}\right)$ if and only if it solves the problem

$$
\text { minimize } J(v) \text { in the set }\{v \in \mathcal{X} \mid v \neq 0, V(v) \geq 0\}
$$

(b) if $G>0$ on $[-\varepsilon, 0) \cup(0, \varepsilon]$, then $u \in \mathcal{X}$ solves ( $\left.\mathcal{P}_{0}^{\prime}\right)$ if and only if it solves the problem

$$
\text { minimize } J(v) \text { in the set }\{v \in \mathcal{X} \mid v \neq 0, V(v) \leq 0\} .
$$

Moreover, any minimizer of $\left(\mathcal{P}_{0}^{\prime \prime}\right)$ or $\left(\mathcal{P}_{0}^{\prime \prime \prime}\right)$ does not change sign.

Proof. It clearly suffices to prove (a).

Consider $v \in \mathcal{X}$ such that $v \geq 0$ a.e. and $V(v)>0$. For $t \geq 0$ we define $v^{t}(x)=$ $\min (v(x), t)$. By (vi) we have $v^{t} \in \mathcal{X}$. We claim that there exists $t_{*}>0$ such that $V\left(v^{t_{*}}\right)=0$.

The continuity of $G$, properties (i) and (iii) in the definition of admissible spaces and the dominated convergence theorem imply that the mapping $t \mapsto V\left(v^{t}\right)=\int_{\mathbf{R}^{N}} G\left(v^{t}(x)\right) d x$ is continuous on $(0, \infty)$. Since $G\left(v^{\varepsilon}(x)\right)<0$ whenever $v(x) \neq 0$ and we cannot have $v(x)=0$ a.e. because $V(v)>0$, we infer that $V\left(v^{\varepsilon}\right)<0$.

We claim that there exists $t_{0}>\varepsilon$ such that $V\left(v^{t_{0}}\right)>0$. Two situations may occur:

Case 1. There exists an increasing sequence $t_{n} \rightarrow \infty$ such that $\left\{G\left(t_{n}\right)\right\}_{n=1}^{\infty}$ is bounded from below. Let $m=\inf _{n \geq 1} G\left(t_{n}\right)$. By dominated convergence we get

$V\left(v^{t_{n}}\right)-V(v)=\int_{\left\{v \geq t_{n}\right\}} G\left(t_{n}\right)-G(v(x)) d x \geq \int_{\left\{v \geq t_{n}\right\}} m-G(v(x)) d x \longrightarrow 0 \quad$ as $n \longrightarrow \infty ;$ hence $V\left(v^{t_{n}}\right) \geq \frac{1}{2} V(v)>0$ for $n$ sufficiently large.

Case 2. $G(s) \longrightarrow-\infty$ as $s \longrightarrow \infty$. Then, since $v \geq 0$ a.e. and $V(v)>0$, we see that the set $A=\{s>0 \mid G(s)>0\}$ is nonempty. Let $M=\sup A<\infty$. It follows that 
$G(s) \leq 0$ for $s \geq M$. It is clear that $M>\varepsilon$ and $V\left(v^{M}\right) \geq V(v)>0$. The claim is thus proved.

Now the continuity of the mapping $t \longmapsto V\left(v^{t}\right)$ implies that there exists $t_{*} \in\left(\varepsilon, t_{0}\right)$ such that $V\left(v^{t_{*}}\right)=0$. Similarly, if $w \in \mathcal{X}, w \leq 0$ a.e. and $V(w)>0$ there is some $\tilde{t}>0$ such that $V\left(-(-w)^{\tilde{t}}\right)=0$.

Next let $u_{0} \in \mathcal{X}$ be a minimizer of $\left(\mathcal{P}_{0}^{\prime}\right)$. Suppose $V(u)>0$ for some $u \in \mathcal{X}$. Then at least one of the quantities $V\left(u_{+}\right)$and $V\left(u_{-}\right)$is positive. If $V\left(u_{+}\right)>0$, take $t_{*}>0$ such that $V\left(u_{+}^{t_{*}}\right)=0$. We have $u_{+}^{t_{*}} \in \mathcal{X} \backslash\{0\}$ and

$$
J(u) \geq J\left(u_{+}\right) \geq J\left(u_{+}^{t_{*}}\right) \geq J\left(u_{0}\right)=T_{0} .
$$

Hence $\inf \{J(u) \mid u \in \mathcal{X}, u \neq 0, V(u) \geq 0\}=J\left(u_{0}\right)=T_{0}$ and $u_{0}$ is a solution of $\left(\mathcal{P}_{0}^{\prime \prime}\right)$.

Conversely, assume that $u$ is a solution of $\left(\mathcal{P}_{0}^{\prime \prime}\right)$. We prove that

$$
V\left(u_{+}\right)=V\left(u_{-}\right)=V(u)=0 .
$$

We argue again by contradiction. If (13) does not hold, the inequality $V\left(u_{+}\right)+V\left(u_{-}\right)=$ $V(u) \geq 0$ implies that at least one of the quantities $V\left(u_{+}\right)$and $V\left(u_{-}\right)$must be positive. Suppose that $V\left(u_{+}\right)>0$. As above we find $t_{*}>0$ such that $V\left(u_{+}^{t_{*}}\right)=0$ and then (12) holds for $u$. Moreover, since $u$ is a minimizer of $\left(\mathcal{P}_{0}^{\prime \prime}\right)$ we have $J(u) \leq T_{0}$ and therefore all inequalities in (12) are in fact equalities. But $J\left(u_{+}\right)=J\left(u_{+}^{t_{*}}\right)$ implies $\int_{\left\{u>t_{*}\right\}}|\nabla u|^{p} d x=0$, hence $\nabla u=0$ a.e. on $\left\{u>t_{*}\right\}$ which gives $\nabla\left(\left(u-t_{*}\right)_{+}\right)=0$ a.e. and we infer that $\left(u-t_{*}\right)_{+}=0$ a.e., that is $u \leq t_{*}$ a.e. Then we have $u_{+}=u_{+}^{t_{*}}$ and consequently $V\left(u_{+}\right)=V\left(u_{+}^{t_{*}}\right)=0$, contrary to our assumption. We argue similarly if $V\left(u_{-}\right)>0$ and (13) is proved. Since $V(u)=0$ and $J(u)=T_{0}=J\left(u_{0}\right)$, we see that $u$ solves $\left(\mathcal{P}_{0}^{\prime}\right)$.

Lastly we show that if $u$ is a minimizer of $\left(\mathcal{P}_{0}^{\prime \prime}\right)$, then either $u_{+}=0$ a.e. or $u_{-}=0$ a.e. (but we cannot have $u_{+}=u_{-}=0$ a.e. because $J(u)=T_{0}>0$ ). Indeed, if $u^{+} \neq 0$ and $u^{-} \neq 0,(13)$ would imply $J\left(u_{+}\right) \geq T_{0}$ and $J\left(u_{-}\right) \geq T_{0}$ and this would give

$$
T_{0}=J(u)=J\left(u_{+}\right)+J\left(u_{-}\right) \geq 2 T_{0}>0,
$$

which is a contradiction. This completes the proof.

Next we prove the monotonicity of scalar minimizers.

Theorem 7 Let $\mathcal{X}$ be an admissible space satisfying the conditions (vi) and (vii). We assume that conditions (C1)-(C3) hold if $1<p<N$, respectively conditions (D1)-(D3) hold if $p=N$. In the case $p=N$, we also assume that there exists $\varepsilon>0$ such that either $G>0$ or $G<0$ on $[-\varepsilon, 0) \cup(0, \varepsilon]$. Then any least energy solution $u$ of $(1)$ such that $\lim _{|x| \rightarrow \infty} u(x)=0$ is, up to a translation, radially symmetric and monotone with respect to $r=|x| \in[0, \infty)$.

Proof. Symmetry follows directly from Propositions 3 and 4. Hence there is a function $\tilde{u}:[0, \infty) \longrightarrow \mathbf{R}$ such that $u(x)=\tilde{u}(|x|)=\tilde{u}(r)$. From Lemma 1 and Proposition 4, we know that any least energy solution is a minimizer of $\left(\mathcal{P}_{\lambda}\right)$ for some $\lambda>0$, respectively of $\left(\mathcal{P}_{0}^{\prime}\right)$. We will show that whenever $u(x)=\tilde{u}(r)$ solves one of these minimization problems and tends to zero at infinity, $\tilde{u}$ is monotone on $[0, \infty)$.

We have to introduce some notation. In what follows, $\mathcal{H}^{N-1}$ is the $(N-1)$-dimensional Hausdorff measure and $\mathcal{L}^{N}$ is the Lebesgue measure on $\mathbf{R}^{N}$. Given a Lebesgue measurable 
set $E \subset \mathbf{R}^{N}$, we denote by $\partial^{*} E$ its measure theoretic boundary, i.e. $\partial^{*} E=\left\{x \in \mathbf{R}^{N} \mid 0<\right.$ $\left.\lim _{r \rightarrow 0} \frac{\mathcal{L}^{N}(E \cap B(x, r))}{\mathcal{L}^{N}(B(x, r))}<1\right\}$. If $E$ has finite measure, we denote by $E^{*}$ the Schwarz rearrangement of $E$, i.e. $E^{*}$ is the open ball centered at the origin such that $\mathcal{L}^{N}(E)=\mathcal{L}^{N}\left(E^{*}\right)$. We recall the isoperimetric inequality: if $E \subset \mathbf{R}^{N}$ is bounded and measurable, then

$$
\mathcal{H}^{N-1}\left(\partial\left(E^{*}\right)\right) \leq \mathcal{H}^{N-1}\left(\partial^{*} E\right),
$$

with equality holding if and only if $E$ is equivalent to a ball in $\mathbf{R}^{N}$ (see, e.g., Proposition 2.2 p. 157 in [6] and references therein). Note that the right side in (14) might be $\infty$.

Now let $u$ be as above. From Proposition 5 and Proposition 6, we know that $u$ has constant sign; hence we may assume that $u \geq 0$. Let $u^{*}$ be the Schwarz rearrangement of $u$. For $t \geq 0$ we denote

$$
E_{t}=\left\{x \in \mathbf{R}^{N} \mid u(x)>t\right\}, \quad F_{t}=\left\{x \in \mathbf{R}^{N} \mid u^{*}(x)>t\right\} .
$$

Note that $E_{t}$ is bounded for any $t>0$ because $u$ tends to zero at $\infty$ and $\left(E_{t}\right)^{*}=F_{t}$.

We argue by contradiction and we assume that $\tilde{u}$ is not nonincreasing. Then there exist $0 \leq r_{1}<r_{2}$ such that $0<\tilde{u}\left(r_{1}\right)<\tilde{u}\left(r_{2}\right)$. Since $\tilde{u}(x) \longrightarrow 0$ as $|x| \longrightarrow \infty$, there exists $r_{3}>r_{2}$ such that $u\left(r_{3}\right)=u\left(r_{1}\right)$. Denoting $a=u\left(r_{1}\right)$ and $b=u\left(r_{2}\right)$, we see that for any $t \in(a, b), E_{t}$ is nonempty and is not equivalent to a ball. The isoperimetric inequality gives

$$
\mathcal{H}^{N-1}\left(\partial F_{t}\right) \leq \mathcal{H}^{N-1}\left(\partial^{*} E_{t}\right) \quad \text { for any } t \in(0, M),
$$

with strict inequality for $t \in(a, b)$.

Since $u \in C^{1}$ and $\left.\lim _{|x| \rightarrow \infty} u \mid x\right)=0$, we see that $u$ is bounded and $(u-t)_{+},\left(u^{*}-t\right)_{+} \in$ $W^{1, p}\left(\mathbf{R}^{N}\right)$ for any $t>0$. Let $M=\max _{x \in \mathbf{R}^{N}} u(x)$. Using the coarea formula for $W^{1, p}$ functions (see, e.g., Proposition 2.1 p. 157 in [6]), we find

$$
\int_{\{u \geq t\}}|\nabla u|^{p} d x=\int_{t}^{M}\left(\int_{u^{-1}(s)}|\nabla u|^{p-1} d \mathcal{H}^{N-1}\right) d s .
$$

The coarea formula for $\left(u^{*}-t\right)+$ gives

$$
\int_{\left\{u^{*} \geq t\right\}}\left|\nabla u^{*}\right|^{p} d x=\int_{t}^{M}\left(\left|\nabla u^{*}\left(\left(u^{*}\right)^{-1}(s)\right)\right|^{p-1} \mathcal{H}^{N-1}\left(\left(u^{*}\right)^{-1}(s)\right)\right) d s .
$$

Passing to the limit as $t \downarrow 0$ and using the monotone convergence theorem, we see that (16) and (17) also hold for $t=0$.

The following result is a simple consequence of Lemma 3.1 p. 161 in [6].

Proposition 8 ([6]) Let $v \in \mathcal{X}$ be a nonnegative function that tends to zero at infinity and let $v^{*}(x)=\tilde{v}^{*}(|x|)$ be the Schwarz rearrangement of $v$. There exists a set $N_{v} \subset$ $(0, \sup (v))$ of Lebesgue measure zero such that for any $t \in(0, \sup (v)) \backslash N_{v},\left(\tilde{v}^{*}\right)^{-1}(t)$ contains only one point, $\left(\tilde{v}^{*}\right)^{\prime}\left(\left(\tilde{v}^{*}\right)^{-1}(t)\right)$ exists, $\mathcal{H}^{N-1}\left(v^{-1}(t)\right)$ and $\mathcal{H}^{N-1}\left(\left(v^{*}\right)^{-1}(t)\right)$ are finite and

$$
\int_{v^{-1}(t)}|\nabla v|^{p-1} d \mathcal{H}^{N-1} \geq\left|\nabla v^{*}\left(\left(v^{*}\right)^{-1}(t)\right)\right|^{p-1} \mathcal{H}^{N-1}\left(\left(v^{*}\right)^{-1}(t)\right) .
$$

Moreover, if $t \in(0, \sup (v)) \backslash N_{v}$ and we have equality in (18) then necessarily

$$
\begin{aligned}
& \mathcal{H}^{N-1}\left(\partial^{*}\left\{x \in \mathbf{R}^{N} \mid v(x)>t\right\}\right)=\mathcal{H}^{N-1}\left(v^{-1}(t)\right)=\mathcal{H}^{N-1}\left(\left(v^{*}\right)^{-1}(t)\right) \\
\text { and }|\nabla v|= & \left|\nabla v^{*}\left(\left(v^{*}\right)^{-1}(t)\right)\right|=\text { constant } \mathcal{H}^{N-1}-\text { a.e. on } v^{-1}(t) .
\end{aligned}
$$


By Proposition 8 we infer that $u$ satisfies (18) for any $t \in(0, M) \backslash N_{u}$, where $\mathcal{L}^{1}\left(N_{u}\right)=$ 0 . Moreover, the isoperimetric inequality (15) (which is strict for $t \in(a, b)$ ) implies that $u$ cannot satisfy (19) for $t \in(a, b) \backslash N_{u}$. Therefore we have strict inequality in (18) for $u$ whenever $t \in(a, b) \backslash N_{u}$. Integrating (18) from 0 to $M$ and using (16) and (17) (with $t=0$ ) we get

$$
\int_{\mathbf{R}^{N}}|\nabla u|^{p} d x>\int_{\mathbf{R}^{N}}\left|\nabla u^{*}\right|^{p} d x \text {, or equivalently } J(u)>J\left(u^{*}\right) .
$$

On the other hand it is clear that $u^{*} \in \mathcal{X} \backslash\{0\}$ and $V\left(u^{*}\right)=V(u)$, therefore $(20)$ contradicts the fact that $u$ is a minimizer. This proves that $\tilde{u}$ must be nonincreasing.

\section{Some remarks and examples}

Remark 9 In the scalar case $m=1$ it is well known (see for example the Introduction of [5]) that if $g$ is odd then any least energy solution has a constant sign. In Remark II.6 of [11], Lions raised the question (for $p=2$ and $N \geq 3$ ) whether this remains true without assuming $g$ odd. Proposition 5 gives an affirmative answer for any $1<p<N$ and Proposition 6, under some mild additional assumptions, for $p=N$. Previous partial results were obtained by Brock [5], using rearrangement arguments, assuming that $1<$ $p \leq 2$, the minimizer $u$ satisfies $u(x) \longrightarrow 0$ as $|x| \rightarrow \infty$ and $g \in C^{0, p-1}(\mathbf{R})$. Nothing was proved for $p>2$.

Remark 10 If $N \geq 3, p=2, m=1$ and under the assumption that $g$ is odd, the existence of least energy solutions for (1) has been proved in [1] by showing that problem $\left(\mathcal{P}_{1}\right)$ admits a minimizer. The minimizer found in [1] was radial by construction, but it was not known whether all least energy solutions were radially symmetric. The existence of a minimizer for $\left(\mathcal{P}_{1}\right)$ without the oddness assumption on $g$ has also been proved in [11], but nothing was known about the symmetry or the sign of such minimizers. Our results imply that any least energy solution is radially symmetric, has constant sign and is monotone with respect to the radial variable, no matter whether $g$ is odd or not.

In the case $N \geq 2, p=2, m \in \mathbf{N}^{*}$, the existence of least energy solutions is also known (see [3] for general results, historical notes, comments and further references). If $N>2$, the existence of a minimizer for $\left(\mathcal{P}_{\lambda}\right)$ and the existence of least energy solutions have been proved in [3] under very general assumptions on the functions $g_{i}$. It has also been shown that the solutions are smooth (Theorem 2.3 p. 105 in [3]) and satisfy the Pohozaev identity (Lemma 2.4 p. 104 in [3]). However, as already mentioned in [3] p. 99 , the existence of radially symmetric least energy solutions was not clear. Indeed, the Schwarz symmetrization that lead to a radial minimizer in [1] could not be used in [3] because of the general assumptions on the nonlinearity made there. In fact, it is known that the Schwarz rearrangements may be used for systems only if the nonlinearity satisfies a cooperative condition.

Proposition 3 above implies that all least energy solutions of the system considered in [3] are radially symmetric.

If $N=2$ and $G(\xi)<0$ for $0<|\xi| \leq \varepsilon$, the existence of least energy solutions and the existence of minimizers for $\left(\mathcal{P}_{0}^{\prime}\right)$ have been proved in $[2,3]$. It has also been shown that such solutions are smooth, satisfy the Pohozaev identity and tend to 0 as $|x| \longrightarrow \infty$. Therefore Proposition 4 implies that any least energy solution is radially symmetric.

We have to mention that if $p=2$ and if the minimizers of $\left(\mathcal{P}_{\lambda}\right)$ satisfy a unique continuation principle, it has already been proved in [12] that any minimizer is radially 
symmetric (modulo translation). In [12] no cooperative condition is required when $m \geq 2$ but using a unique continuation principle require in particular $g$ to be $C^{1}$. Our results are still valid when a unique continuation principle fails (e.g., for minimizers with compact support). Note that compactly supported minimizers may occur in some applications (cf. Theorem 3.2 (ii) p. 111 in [3]; see also [14] for such an example). In the scalar case $m=1,[12]$ does not say anything about the sign of the minimizers.

Remark 11 If $1<p<N$ and $m=1$, it has been proved in [9], under general conditions on $g$, that problem $\left(\mathcal{P}_{\lambda}\right)$ admits minimizers (thus (1) has least energy solutions). The minimizers found in [9] were radially symmetric by construction. It follows from Proposition 3 that any least energy solution is radially symmetric.

If, in addition to the assumptions of Theorem 7, it is assumed that $g$ is locally Lipschitz on $(0, \infty)$ and non-increasing on some interval $\left(0, s_{0}\right)$ and $1<p<2$, it has been proved in [8] that any nonnegative solution of (1) is radially symmetric and that $u(x)=\tilde{u}(|x|)$ satisfies $\tilde{u}^{\prime}(r)<0$ whenever $r>0$ and $\tilde{u}(r)>0$. The same result is true when $p>2$ if it is assumed in addition that the critical set of the solution $u$ is reduced to one point (see [15]). These assumptions are not necessary for us but, of course, we only deal with least energy solutions.

Remark 12 (i) The symmetry results in Section 2 hold without any change if we replace the functional $J$ by a functional of the form $\int_{\mathbf{R}^{N}} \sum_{i=1}^{m} A_{i}\left(u, \nabla u_{i}\right) d x$ where $\xi \rightarrow A_{i}(u, \xi)$ is $p$-homogeneous for any $i=1, \ldots, m$.

(ii) Our method still works for more general functionals of the form

$$
\tilde{J}(u)=\frac{1}{p} \int_{\mathbf{R}^{N}}|x|^{\alpha} \sum_{i=1}^{m} A_{i}(u)\left|\nabla u_{i}\right|^{p} d x \quad \text { and } \quad \tilde{V}(u)=\int_{\mathbf{R}^{N}}|x|^{\beta} G(u) d x .
$$

In this case, using Theorem 1 in [14], we obtain that minimizers (and the corresponding minimum action solutions) are axially symmetric.

Functionals of this type appear, e.g., in the Caffarelli-Kohn-Nirenberg problem (which consists in minimizing $\int_{\mathbf{R}^{N}}|\nabla u|^{q}|x|^{-a q} d x$ under the constraint $\int_{\mathbf{R}^{N}}|u|^{p}|x|^{-b p} d x=$ const., where $q>1, p>1, a \leq b<\frac{N}{q}$ and $\left.0<\frac{1}{q}-\frac{1}{p}=\frac{1+a-b}{N}\right)$. It has been proved that minimizers for this problem exist and, in general, are not radially symmetric (see [4] and references therein).

Acknowledgment The research of the first author was supported in part by KRF2007-412-J02301 of Korea Research Foundation.

\section{References}

[1] H. Berestycki, P.-L. Lions, Nonlinear scalar field equations, I. Existence of a ground state, Arch. Rational Mech. Anal. 82 (1983), 313-345.

[2] H. Berestycki, T. Gallouët, O. Kavian, Equations de champs scalaires euclidens non linéaires dans le plan, C.R. Acad. Sc. Paris Série I - Math. 297 (1983), 307-310 and Publications du Laboratoire d'Analyse Numérique, Université de Paris VI, 1984.

[3] H. Brézis, E. H. Lieb, Minimum Action Solutions for Some Vector Field Equations, Comm. Math. Phys. 96 (1984), 97-113. 
[4] J. Byeon, Z.-Q. WAng, Symmetry breaking of extremal functions for the CaffarelliKohn-Nirenberg inequalities, Comm. Contemp. Math. 4 (2002), 457-465.

[5] F. Brock, Positivity and radial symmetry of solutions to some variational problems in $\mathbf{R}^{N}$, J. Math. Anal. Appl. 296 (2004), 226-243.

[6] J. E. Brothers, W.P. Ziemer, Minimal rearrangements of Sobolev functions, J. reine angew. Math. 384 (1988), 153-179.

[7] J. Busca, B. Sirakov, Symmetry results for semilinear elliptic systems in the whole space, J. Diff. Eq. 163, No. 1 (2000), 41-56.

[8] L. Damascelli, F. Pacella, M. Ramaswamy, Symmetry of ground states of p-Laplace equations via the moving plane method, Arch. Rational Mech. Anal. 148 (1999), 291-308.

[9] A. Ferrero, F. Gazzola, On subcriticality assumptions for the existence of ground states of quasilinear elliptic equations, Adv. Diff. Eq. 8, No. 9 (2003), 1081-1106.

[10] B. Gidas, W. N. Ni, L. NiREnBerg, Symmetry of positive solutions of nonlinear elliptic equations in $\mathbf{R}^{n}$, Adv. Math. Supp. Stud. 7A (1981), 369-403.

[11] P.L. Lions, The concentration-compactness principle in the Calculus of Variations, The locally compact case, Part 2, Ann. Inst. H. Poincaré Anal. Non Linéaire 1 (1984), 223-283.

[12] O. LOPES, Radial symmetry of minimizers for some translation and rotation invariant functionals, J. Diff. Eq. 124 (1996), 378-388.

[13] O. Lopes, M. Montenegro, Symmetry of mountain pass solutions for some vector field equations, J. Dyn. Diff. Eq. 18, No. 4 (2006), 991-999.

[14] M. MARIŞ, On the symmetry of minimizers, Arch. Rational Mech. Anal., to appear; arXiv:0712.3386 (www.arxiv.org)

[15] J. Serrin, H. Zou, Symmetry of ground states of quasilinear elliptic equations, Arch. Rational Mech. Anal. 148 (1999), 265-290. 